\title{
The Plot Thickens
}

The approach to clinical conundrums by an expert clinician is revealed through the presentation of an actual patient's case in an approach typical of a morning report. Similarly to patient care, sequential pieces of information are provided to the clinician, who is unfamiliar with the case. The focus is on the thought processes of both the clinical team caring for the patient and the discussant.

This icon represents the patient's case. Each paragraph that follows represents the discussant's thoughts.

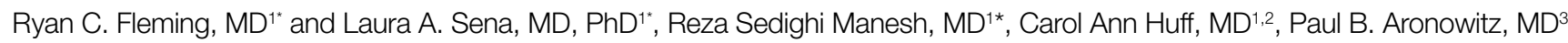

${ }^{1}$ Department of Medicine and ${ }^{2}$ Department of Oncology, Johns Hopkins University School of Medicine, Baltimore, Maryland; ${ }^{3}$ Department of Internal Medicine, University of California Davis Medical Center, Sacramento, California. *Both authors contributed equally to this work.

After losing consciousness at a supermarket, a 70-yearold man was brought to the emergency department by paramedics. He subsequently developed chest pain.

Syncope can be difficult to evaluate, but chest pain may help narrow an otherwise broad differential diagnosis. If this patient has aortic stenosis or hypertrophic cardiomyopathy, effort syncope is the culprit. Cardiac dysrhythmia (eg, ventricular tachycardia), complete heart block, and supraventricular tachycardia each can cause syncope along with chest pain. Myocardial infarction and associated ventricular arrhythmia might also explain both chest pain and syncope. The paramedics might have noted an arrhythmia on the cardiac monitor; if possible, the rhythm strip should be reviewed. A pulmonary embolus can cause chest pain and, if large enough to cause right ventricular compromise, syncope.

According to witnesses at the supermarket, the patient dropped to the ground, lost consciousness, and convulsed for 30 seconds. He had no head trauma, tongue biting, urinary incontinence, or confusion afterward. Electrocardiogram (ECG) performed at the scene showed ST elevations in leads V1 to V3 with ST depressions in the inferior leads. On arrival in the emergency department, the patient described nonradiating substernal chest pressure exacerbated by deep inhalation. The pain did not improve with nitroglycerin. $\mathrm{He}$ recalled feeling light-headed before the syncope.

He had not received medical care for 20 years and had no known illnesses other than hypertension. He was not taking any medications. He previously worked as a welder and never smoked tobacco, drank alcohol, or used illicit drugs.

\footnotetext{
*Address for correspondence and reprint requests: Reza Sedighi Manesh, $\mathrm{MD}$, Division of General Internal Medicine, Johns Hopkins University School of Medicine, 600 N Wolfe St, Nelson 215, Baltimore, MD 21287; Telephone: 412708-6944; Fax: 410-502-0923; E-mail: rsedigh1@jhmi.edu
}

Received: April 13, 2016; Revised: September 26, 2016; Accepted: October 5, 2016

2017 Society of Hospital Medicine DOI 10.12788/jhm.2775
The patient's temperature was $36.4^{\circ} \mathrm{C}$. Heart rate was 88 beats per minute, blood pressure $128 / 72 \mathrm{~mm} \mathrm{Hg}$, oxygen saturation $100 \%$ on room air, and respiratory rate 22 breaths per minute. The patient had conjunctival pallor. There was a grade $3 / 6$ crescendo-decrescendo systolic murmur loudest at the right upper sternal border without radiation to the carotids. There was no jugular venous distention. Lungs were clear to auscultation bilaterally. There was no peripheral edema, rash, or lymphadenopathy.

Convulsive movements commonly occur during episodes of unconsciousness lasting more than 15 seconds-a phenomenon termed convulsive syncope and often is confused with seizures. These movements are usually clonic jerks of the extremities and trunk and slight twitching of the face, and occasionally tonic extension of the trunk and clenching of the jaw. Absence of tongue biting, urinary incontinence, and confusion in this patient's case makes seizures less likely.

The distribution of ST segment changes on his ECG are concerning for myocardial infarction in the septal and inferior regions. Right-sided ECG should be performed to assess for right ventricular infarction. Although myocardial ischemia is the primary concern, some features warrant consideration of other etiologies of syncope. First, syncope is an unusual presentation of cardiac ischemia or infarct. The complaint of chest pressure exacerbated by deep inhalation is another atypical feature for myocardial ischemia. Although the patient's oxygen saturation and heart rate are normal, pulmonary embolism remains a possibility.

The prominent crescendo-decrescendo systolic murmur at the right upper sternal border could indicate aortic stenosis; the carotids should be palpated to assess for pulsus parvus et tardus. A high-flow state associated with anemia could also lead to a midsystolic murmur. Conjunctival pallor typically is seen with hemoglobin levels of $6 \mathrm{~g} / \mathrm{dL}$ or less. This finding may indicate severe anemia, which has the potential to cause myocardial ischemia and syncope.

Laboratory testing revealed a troponin of $0.04 \mathrm{ng} / \mathrm{dL}$, hemoglobin $4.1 \mathrm{~g} / \mathrm{dL}$ with MCV of $84.7 \mathrm{fL}$, white blood cell count $6,500 / \mu \mathrm{L}$ and platelet count $179,000 / \mu \mathrm{L}$. 
Serum sodium was $130 \mathrm{mEq} / \mathrm{L}$, urea nitrogen $16 \mathrm{mg} / \mathrm{dL}$, creatinine $1.6 \mathrm{mg} / \mathrm{dL}$, calcium $7.8 \mathrm{mg} / \mathrm{dL}$, total protein $11.4 \mathrm{~g} / \mathrm{dL}$ (reference range, 6.0-8.2), and albumin $2.2 \mathrm{~g} /$ dL. Erythrocyte sedimentation rate (ESR) was $20 \mathrm{~mm} / \mathrm{h}$. Serum iron was $48 \mu \mathrm{g} / \mathrm{mL}$, total iron binding capacity 275 $\mu \mathrm{g} / \mathrm{dL}$, percent iron saturation $17 \%$ (reference range, 20. 55), and ferritin $10 \mathrm{ng} / \mathrm{mL}$ (reference range, 30-400). The international normalized ratio (INR) was 1.5 , prothrombin time $15.5 \mathrm{sec}$ (reference range, 9.4-11.6), and partial thromboplastin time $24.7 \mathrm{sec}$ (reference range, 22.9-30.6). Hepatitis $\mathrm{C}$ and $\mathrm{HIV}$ antibodies were negative as was the urine toxicology screen. Urine protein to creatinine ratio was 0.07 . His hemoglobin rose to $7.9 \mathrm{~g} / \mathrm{dL}$ with transfusion of 4 units packed red blood cells (RBC). His chest pain improved and inferior ST depressions resolved on follow-up ECG. Further history revealed multiple episodes of melena and hematochezia in the preceding weeks without nausea, vomiting, or abdominal pain.

The patient has a strikingly large gamma gap: 9.2. A gap larger than 4 is concerning for the presence of paraproteins. Given the possibility of a paraproteinemia (eg, multiple myeloma, plasmacytoma, Waldenström macroglobulinemia), the first step is to check serum and urine protein electrophoresis. The patient's anemia is significant and reticulocyte index low. The low ferritin level combined with the inappropriately low reticulocyte count could result from iron deficiency anemia, another bone marrow process, or both. The patient's syncope likely resulted from severe anemia and hypovolemia associated with hematochezia. The prolonged prothrombin time could be caused by a coagulation factor production problem, from vitamin $\mathrm{K}$ deficiency or underlying liver dis-

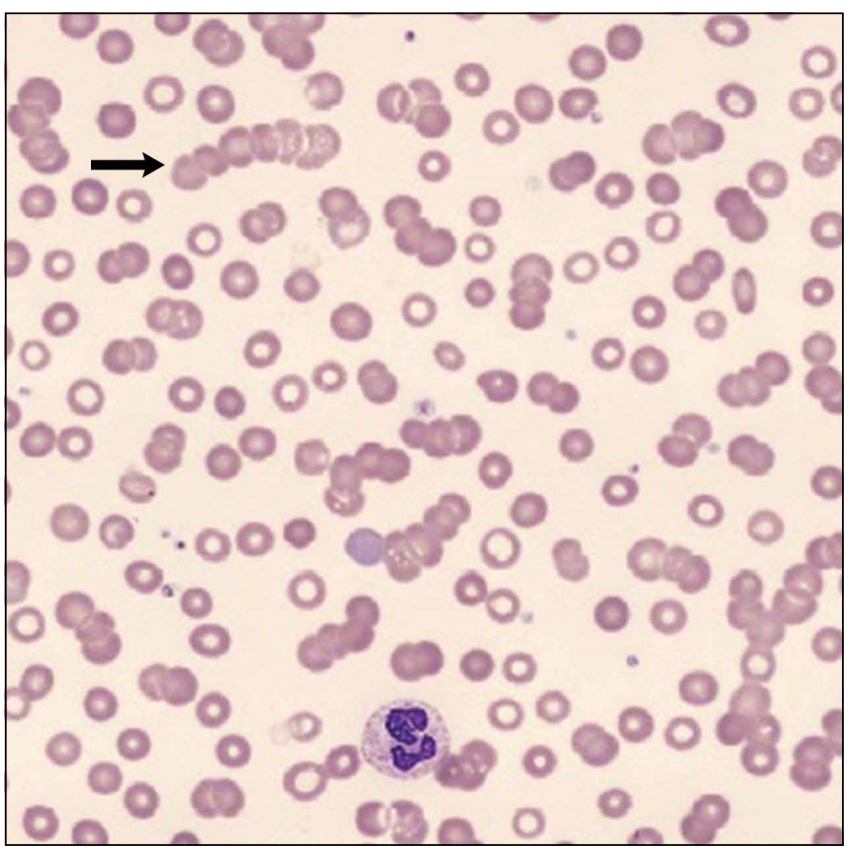

FIG 1. Patient's peripheral blood smear shows moderate rouleaux formation (arrow) and increased background staining secondary to hypergammaglobulinemia. ease, or by a consumptive problem, from low-grade disseminated intravascular coagulation. It is controversial whether inhaling welding fumes causes cancer, but the patient's age alone makes malignancy a definite possibility.

The patient was admitted to the progressive care unit with a plan for urgent endoscopy with cardiac anesthesia. Troponin level peaked at $0.84 \mathrm{ng} / \mathrm{dL} 10$ hours after admission. Transthoracic echocardiogram revealed ejection fraction $40 \%$ with pseudo-normal filling pattern, right ventricular systolic blood pressure 60 to $65 \mathrm{~mm} \mathrm{Hg}$, and echodense material with overlying spontaneous echo contrast leading into the right atrium (a concern for thrombus or tumor); diffuse sclerosis and mild stenosis of the aortic valve were seen with mild to moderate tricuspid regurgitation. Intravenous-contrast computed tomography (CT) of chest, abdomen, and pelvis showed descending and sigmoid colon diverticulosis and an area of circumferential wall thickening of the rectum and anus with adjacent perirectal lymph nodes and inflammatory stranding. There was no evidence of pulmonary embolism.

Aortic stenosis is intriguing in light of the patient's painless melena and hematochezia. Heyde syndrome is a phenomenon in which high shear stress causes a reduction in the size of von Willebrand factor predisposing to bleeding from submucosal angiodysplasia. However, Heyde syndrome has been reported to occur in the setting of severe or critical aortic stenosis and is unlikely to be the cause here. The patient needs to be examined with both upper and lower endoscopy to rule out gastrointestinal (GI) malignancy, gastric and esophageal varices, and painless peptic ulcers. High right ventricular systolic blood pressures along with echodense material in the inferior vena cava and right atrium suggest the possibility of malignancy with vascular invasion. Tricuspid regurgitation is consistent with high right-sided pressures. As left ventricular ejection fraction is reduced, some of the high right-sided pressures could also be attributable to left heart failure. CT findings of rectal wall thickening and perirectal lymph nodes could be attributable to cancer with locally metastatic disease. Blood loss caused by this cancer would explain the severe anemia on admission as well as the low ferritin level and the iron deficiency anemia. In this 70-year-old man who has not had routine health care maintenance, the leading diagnosis is colorectal cancer. However, the markedly elevated globulin gap and elevated INR strongly suggest another process (eg, multiple myeloma, other paraproteinemia) is also present.

INR remained elevated (1.5) despite vitamin K supplementation. Peripheral blood smear showed hypochromic and normocytic RBCs with moderate rouleaux formation (Figure 1). Intravenous pantoprazole and octreotide were started. Upper and lower endoscopy revealed multiple esophageal erosions and both a polypoid mass and an ulcer within the rectum with evidence of prior bleeding. The mass was resected and the ulcer biopsied. 

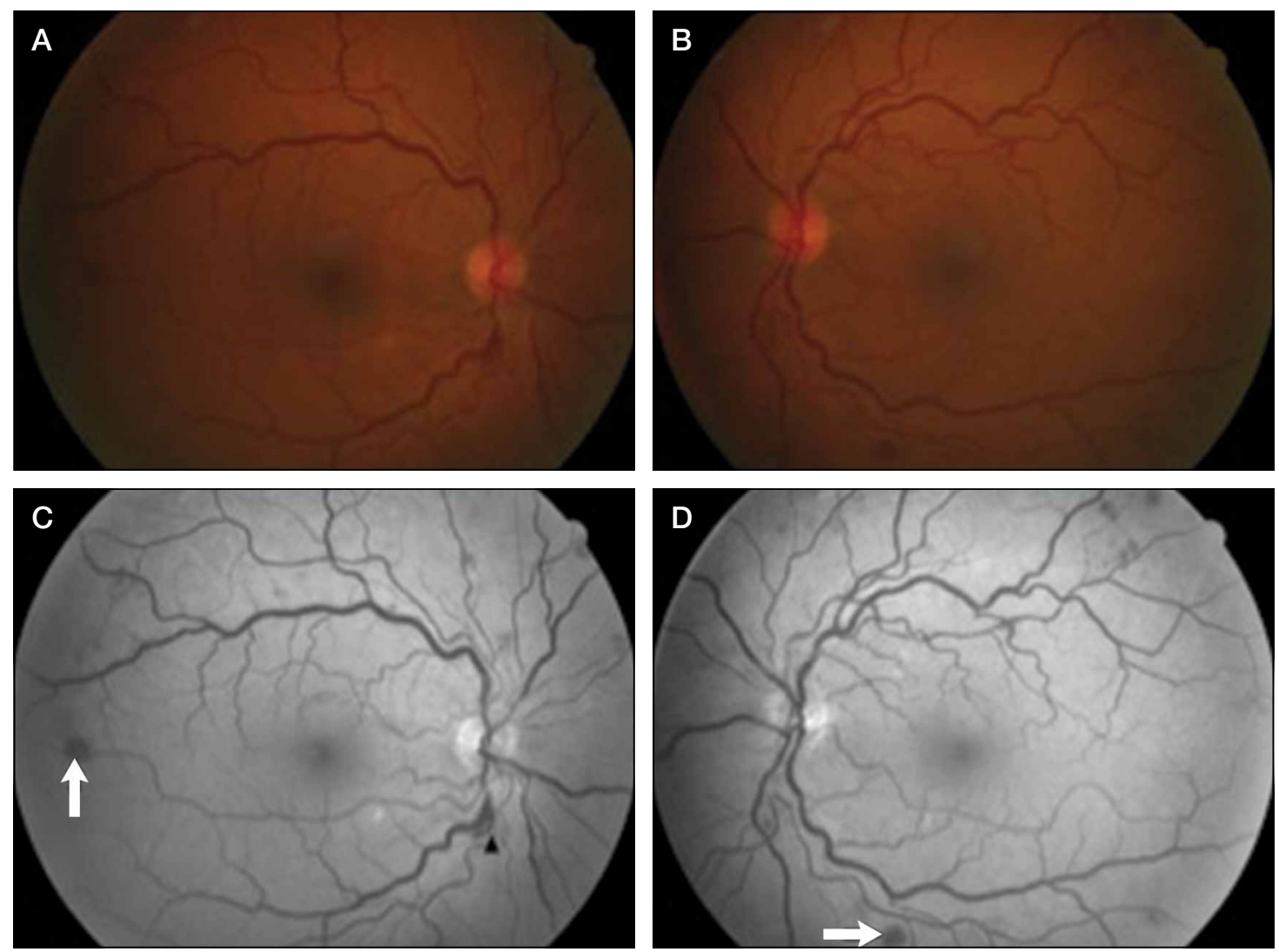

FIG 2. (A,B) Color fundus photographs show mild dilatation of patient's retinal veins and retinal hemorrhages. (C,D) Red-free fundus photographs show round blot hemorrhages occurring from vessels within retina (arrow) and linear, flame-shaped hemorrhages occurring from larger vessels at retinal surface, spreading between ganglion cell axons and resulting in linear appearance (arrowhead).

On hospital day 3, the patient was transfused another unit of packed RBCs. Hemoglobin level increased from $7.4 \mathrm{~g} / \mathrm{dL}$ to $8.2 \mathrm{~g} / \mathrm{dL}$, but he began to complain of headache, blurred vision, and worsened chest pain. He did not have weakness, numbness, diplopia, dysphagia, or dysarthria. External examination and extraocular movements of both eyes were normal. Visual acuity was $20 / 25$ bilaterally. Funduscopic examination revealed mild dilation of retinal veins and retinal hemorrhages (Figure 2).

Rouleaux formation occurs as excess cathodal proteins, such as immunoglobulins or fibrinogen, adhere to RBCs and cause the cells to stack together in long chains. Classically this is associated with multiple myeloma, but can occur with Waldenström's macroglobulinemia and other cancers or infections. It can also occur as an artifact in smear preparation but the large globulin gap in this patient supports pathologic rouleaux formation.

Venous retinopathy with hemorrhages may occur with occlusion of the arterial supply (eg, as with carotid artery obstruction) but also with hyperviscosity syndrome (HVS). Vascular disturbances throughout the body play a major role in HVS, but these changes are most easily visualized in the retina. It is interesting that the patient's headache and blurred vision began after he received additional blood transfusions.
Spuriously low hemoglobin and hematocrit levels may stem from increased plasma volume from high immunoglobulin $\mathrm{M}$ (IgM) concentrations in Waldenström macroglobulinemia; thus, RBC transfusions can exacerbate symptoms by elevating total RBC mass. Normocytic, normochromic anemia is characteristic of both multiple myeloma and Waldenström's macroglobulinemia. That the patient's chest pain recurred coincidentally with blurred vision and headache suggests the likely cause is cardiac ischemia from hyperviscosity. The serum viscosity level should be checked, and, if it is elevated, urgent serum plasmapheresis should be considered. Determining the source of excess globulin production and treating the underlying disease are crucial at this juncture.

In the general population, rectal adenocarcinoma is the most common cause of a rectal mass. In this patient, presence of a paraproteinemia may point to a different diagnosis. Extramedullary colorectal plasmacytoma can occur in the rectum but is exceedingly rare. Waldenström's macroglobulinemia, a subtype of lymphoplasmacytic lymphoma, can be associated with a rectal lymphoma. At this point, it is not possible to confidently predict the etiology of the mass.

Plasma viscosity was elevated at 4.0 (reference range, 1.6-1.9). Serum protein electrophoresis revealed an $\mathrm{M}$-spike of $6.4 \mathrm{~g} / \mathrm{dL}$ corresponding to $\mathrm{IgG}$ on immunofixa- 


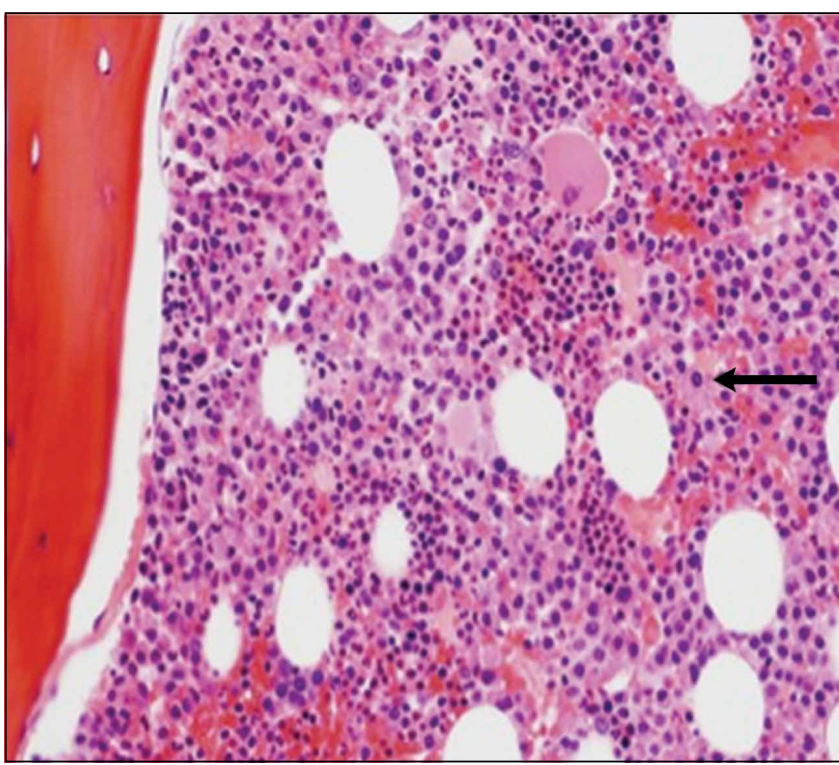

FIG 3. Patient's bone marrow biopsy results revealed hypercellular bone marrow with morphologically mature plasma cells (arrow).

tion. The kappa/lambda light chain ratio was 2.2 with normal urine protein electrophoresis. Bone marrow core biopsy demonstrated 60 to $70 \%$ plasma cells (Figure 3) with aspirate flow cytometry showing $3 \%$ phenotypically abnormal monoclonal plasma cells that were kappa positive. Skeletal survey revealed possible small lytic lesions in right scapula and proximal humeri bilaterally. Hematoxylin and eosin stain of rectal ulcer was highly suggestive of amyloidosis. Pathology of the polypoid mass was consistent with at least high-grade dysplasia arising in a tubular adenoma. The initial colonoscopy was limited by poor colonic preparation. A sigmoidoscopy with biopsy 6 weeks later revealed a $4 \mathrm{~cm}$ rectal mass, which pathology showed moderately to poorly infiltrating adenocarcinoma with necrosis (clinical stage $\mathrm{T} 3 \mathrm{~N} 1$ ).

He was started on cyclophosphamide, bortezomib and dexamethasone for $\operatorname{IgG} \kappa$ myeloma with improvement in his headache, blurred vision, chest pain, and plasma viscosity (4 to 1.8 ). His hemoglobin remained stable at $10 \mathrm{~g} /$ dL. Neoadjuvant Capecitabine and radiation therapy were initiated for his rectal cancer.

\section{DISCUSSION}

Multiple myeloma is characterized by monoclonal proliferation of plasma cells, elevated circulating monoclonal immunoglobulin, and end-organ damage. ${ }^{1}$ It accounts for approximately $0.8 \%$ of all new cancer diagnoses; average age at onset is 70 years. The patient described here had an unusual presentation, with GI bleeding and progression to HVS, and known risk factors for multiple myeloma (male sex, low socioeconomic status, welding career). ${ }^{2,3}$

An early clue in the diagnosis was the patient's large gamma gap and concurrent anemia. Gamma gap, calculated by subtracting serum albumin from serum total protein, is so named because it often reflects an elevated gamma globulin concentration. However, it actually reflects all nonalbumin serum protein. A gamma gap larger than $3.1 \mathrm{~g} / \mathrm{dL}$ is an independent risk factor for death ${ }^{4}$ and may be associated with infection, autoimmunity, and malignancy. Although there are no screening guidelines for multiple myeloma, $73 \%$ of cases are brought to attention by anemia discovered on routine laboratory investigation. ${ }^{5}$ This patient's lack of prior medical care likely contributed to his atypical presentation. Screening colonoscopy, recommended at age 50, might have identified his rectal cancer at an earlier stage.

The patient's anemia was likely secondary to GI hemorrhage and bone marrow suppression. His hematochezia might have been partly related to the pathophysiologic interaction of paraproteins with platelets, coagulation factors, and blood vessels. ${ }^{6}$ Amyloidosis of the GI tract is seen in $8 \%$ of AL amyloidosis ${ }^{7}$ and most frequently manifests as gastrointestinal bleeding, which is thought to be due to ischemia, vascular friability, or mucosal lesions. It less commonly presents as malabsorption or dysmotility. ${ }^{8}$ Although gastrointestinal amyloid is not typically associated with radiologic abnormalities, occasionally it may cause luminal wall thickening, adenopathy, and inflammatory stranding. ${ }^{9}$ The gold standard for diagnosis is tissue biopsy. However, presence of amyloidosis does not change the overall treatment strategy for multiple myeloma.

An interesting feature of this case is the development of HVS, which typically manifests with mucosal bleeding, blurred vision, and headache. ${ }^{10}$ HVS can be diagnosed on retinal examination with findings of venous tortuosity, dilatation, and intraretinal hemorrhage, as occurred in this case,$^{11}$ and is confirmed with serum viscosity measurement. The first evidence of HVS in this case might have been the spontaneous echo contrast, or "smoke," detected on echocardiogram. Spontaneous echo contrast represents increased $\mathrm{RBC}$ aggregation, from interaction of RBCs and plasma proteins, at low shear rates, ${ }^{12}$ and is associated with conditions that result in left atrial stasis, such as atrial arrhythmias and mitral stenosis. This patient did not have valvular pathology or arrhythmia, and thus the "smoke" likely reflected HVS.

Of the paraproteinemias, Waldenström's macroglobulinemia is most often associated with HVS, likely because of the pentameric structure of $\operatorname{IgM}^{13}$ and the consequential large size that predisposes to vascular occlusion. Whereas HVS can occur with IgM levels as low as $3 \mathrm{~g} / \mathrm{dL}$, it typically does not occur with $\mathrm{IgG}$ concentrations under $15 \mathrm{~g} / \mathrm{dL}$. This patient presented with an IgG level of $8 \mathrm{~g} / \mathrm{dL}$ and developed HVS symptoms only after multiple packed RBC transfusions. Elevated IgG level likely made him susceptible to HVS, which ultimately was precipitated by blood transfusion. Therefore, this patient's initial chest pain most likely was caused by demand cardiac ischemia secondary to anemia, whereas his subsequent, posttransfusion chest pain likely resulted from hyperviscosity angina. Hyperviscosity angina — cardiac ischemia resulting from poor coronary perfusion caused by hyperviscous blood-has been described in polycythemia and connective tissue disorders. ${ }^{14,15}$ To our knowledge, however, hyperviscosity angina 
has not been reported in patients with multiple myeloma. Treatment of hyperviscosity with end-organ damage typically consists of plasmapheresis, but this patient was started on urgent chemotherapy, and his symptoms improved. Untreated HVS can lead to end-organ ischemia and death.

This patient had a multitude of seemingly disparate symptoms and abnormalities that ultimately were united in a diagnosis of IgG $\kappa$ multiple myeloma. Subsequently diagnosed rectal adenocarcinoma may have led to ongoing blood loss, which worsened the anemia, but had no evident relation to the primary diagnosis of multiple myeloma. This case exemplifies the fact that HVS is a rare but important iatrogenic complication of multiple myeloma treated with blood transfusion. As this patient's hospital course progressed, the plot, and his blood, thickened.

\section{KEY TEACHING POINTS}

- Multiple myeloma is occasionally associated with HVS, which manifests with mucosal bleeding, blurred vision, and headache.

- Hyperviscosity angina—cardiac ischemia resulting from

\section{References}

1. Palumbo A, Anderson K. Multiple myeloma. N Engl J Med. 2011;364(11):1046-1060.

2. Koessel SL, Theis MK, Vaughan TL, et al. Socioeconomic status and the incidence of multiple myeloma. Epidemiology. 1996;7(1):4-8.

3. Fritschi L, Siemiatycki J. Lymphoma, myeloma and occupation: results of a case-control study. Int J Cancer. 1996;67(4):498-503.

4. Juraschek SP, Moliterno AR, Checkley W, Miller ER 3rd. The gamma gap and allcause mortality. PLoS One. 2015;10(12):e0143494.

5. Kyle RA, Gertz MA, Witzig TE, et al. Review of 1027 patients with newly diagnosed multiple myeloma. Mayo Clin Proc. 2003;78(1):21-33.

6. Eby CS. Bleeding and thrombosis risks in plasma cell dyscrasias. Hematology Am Soc Hematol Educ Program. 2007:158-164.

7. Menke DM, Kyle RA, Fleming CR, Wolfe JT 3rd, Kurtin PJ, Oldenburg WA. Symptomatic gastric amyloidosis in patients with primary systemic amyloidosis. Mayo Clin Proc. 1993;68(8):763-767.

8. Levy DJ, Franklin GO, Rosenthal WS. Gastrointestinal bleeding and amyloidosis. Am J Gastroenterol. 1982;77(6):422-426. poor coronary perfusion caused by hyperviscous bloodshould be considered in patients with paraproteinemias and chest pain.

- Plasmapheresis reverses the clinical manifestations of HVS but not the underlying disease process (eg, Waldenström's macroglobulinemia, multiple myeloma, leukemia, polycythemia).

- Spontaneous echo contrast represents increased RBC aggregation, from interaction of RBCs and plasma proteins, at low shear rates, and is associated with left atrial stasis, commonly from atrial fibrillation or mitral stenosis, but might be present in HVS.

\section{Acknowledgment}

The authors thank Peter Campochiaro, MD, and Whitney Green, MD, for their contributions to the images used in this article.

Disclosure: Dr. Sedighi Manesh is supported by the Jeremiah A. Barondess Fellowship in the Clinical Transaction of the New York Academy of Medicine, in collaboration with the Accreditation Council for Graduate Medical Education (ACGME).

The other authors have nothing to report.

9. Araoz PA, Batts KP, MacCarty RL. Amyloidosis of the alimentary canal: radiologic-pathologic correlation of CT findings. Abdom Imaging. 2000;25(1):38-44.

10. Stone MJ, Bogen SA. Evidence-based focused review of management of hyperviscosity syndrome. Blood. 2012;119(10):2205-2208.

11. Rajagopal R, Apte RS. Seeing through thick and through thin: retinal manifestations of thrombophilic and hyperviscosity syndromes. Surv Ophthalmol. 2016;61(2):236-247

12. Black IW. Spontaneous echo contrast: where there's smoke there's fire. Echocardiography. 2000;17(4):373-382.

13. Kwaan HC. Hyperviscosity in plasma cell dyscrasias. Clin Hemorheol Microcirc. 2013;55(1):75-83.

14. Piccirillo G, Fimognari FL, Valdivia JL, Marigliano V. Effects of phlebotomy on a patient with secondary polycythemia and angina pectoris. Int $\mathrm{J}$ Cardiol. 1994;44(2):175-177.

15. Ovadia S, Lysyy L, Floru S. Emergency plasmapheresis for unstable angina in a patient with hyperviscosity syndrome. Am J Emerg Med. 2005;23(6):811-812. 